\title{
A characterization of the Burr Type III and Type XII distributions through the method of percentiles and the Spearman correlation
}

\section{Mohan D. Pant \& Todd C. Headrick}

To cite this article: Mohan D. Pant \& Todd C. Headrick (2017) A characterization of the Burr Type III and Type XII distributions through the method of percentiles and the Spearman correlation, Communications in Statistics - Simulation and Computation, 46:2, 1611-1627, DOI: 10.1080/03610918.2015.1048878

To link to this article: http://dx.doi.org/10.1080/03610918.2015.1048878

Accepted author version posted online: 30

Jun 2015.

Published online: 30 Jun 2015.

Submit your article to this journal $\widetilde{ }$

Џ Article views: 177

Q View related articles $\sqsubset$

View Crossmark data \lceil

4 Citing articles: 1 View citing articles 준 


\title{
A characterization of the Burr Type III and Type XII distributions through the method of percentiles and the Spearman correlation
}

\author{
Mohan D. Pant ${ }^{\mathrm{a}}$ and Todd C. Headrick ${ }^{\mathrm{b}}$ \\ a Department of Curriculum and Instruction, University of Texas at Arlington, Arlington, Texas, USA; ${ }^{b}$ Section in \\ Quantitative Methods, Department of CQMSE, Southern Illinois University Carbondale, Carbondale, Illinois, USA
}

\begin{abstract}
A characterization of Burr Type III and Type XII distributions based on the method of percentiles (MOP) is introduced and contrasted with the method of (conventional) moments (MOM) in the context of estimation and fitting theoretical and empirical distributions. The methodology is based on simulating the Burr Type III and Type XII distributions with specified values of medians, inter-decile ranges, left-right tail-weight ratios, tail-weight factors, and Spearman correlations. Simulation results demonstrate that the MOP-based Burr Type III and Type XII distributions are substantially superior to their (conventional) MOM-based counterparts in terms of relative bias and relative efficiency.
\end{abstract}

\section{ARTICLE HISTORY}

Received 20 August 2014

Accepted 1 May 2015

\section{KEYWORDS}

Method of Moments; Method of Percentiles; Monte Carlo Simulation

\section{MATHEMATICS SUBJECT} CLASSIFICATION Primary 62E17, 62F40, 62G05, $62 \mathrm{G} 09,62 \mathrm{H} 05,62 \mathrm{H} 10,62 \mathrm{H} 12$, $62 \mathrm{H} 20$; Secondary $65 \mathrm{C} 05$

\section{Introduction}

Of the 12 distribution functions introduced by Burr (1942), the Burr Type III and Type XII distributions have received much of the attention because these include several families of non-normal distributions (e.g., the Gamma distribution) with varying degrees of skew and kurtosis (Burr, 1973; Headrick et al., 2010; Rodriguez, 1977; Tadikamalla, 1980). These distributions have been used primarily for statistical modeling of events arising in a variety of applied mathematical contexts. Some examples of such applications include modeling events associated with forestry (Gove et al., 2008; Lindsay et al., 1996), fracture roughness (Nadarajah and Kotz, 2006, 2007), life testing (Wingo, 1983, 1993), operational risk (Chernobai et al., 2007), option market price distributions (Sherrick et al., 1996), meteorology (Mielke, 1973), modeling crop prices (Tejeda and Goodwin, 2008), software reliability growth (Abdel-Ghaly et al., 1997), reliability analysis (Mokhlis, 2005), and in the context of Monte Carlo simulation studies (Headrick et al., 2010; Pant and Headrick, 2013).

The cumulative distribution functions (cdfs) associated with the Burr Type III and Type XII distributions are given as (Burr, 1942) Eqs. (11) and (20),

$$
\begin{aligned}
F(x)_{\mathrm{III}} & =\left(1+x^{c}\right)^{-k}, \\
F(x)_{\mathrm{XII}} & =1-\left(1+x^{c}\right)^{-k},
\end{aligned}
$$

CONTACT Mohan D.Pant mpant@uta.edu EDepartment of Curriculum and Instruction, University of Texas at Arlington, 320-B Science Hall, Arlington, TX 76019, USA.

Color versions of one or more of the figures in the article can be found online at www.tandfonline.com/lssp.

() 2017 Taylor \& Francis Group, LLC 
where $x \in(0, \infty)$, and $c$ and $k$ are real-valued shape parameters that are also used to compute the values of mean and standard deviation of a distribution. Parameter $c$ is negative for Type III and positive for Type XII distribution, whereas parameter $k$ is positive for both Type III and Type XII distributions (Headrick et al., 2010). The scale parameter that is typically used to characterize Burr distributions (e.g., Gove et al., 2008; Lindsay et al., 1996; Tadikamalla, 1980) is assumed to be one in (1) and (2) as in Burr (1942).

The quantile functions associated with (1) and (2) are expressed as (Headrick et al., 2010) Eqs. (5) and (6),

$$
\begin{aligned}
q(u)_{\mathrm{III}} & =\left(u^{-1 / k}-1\right)^{1 / c}, \\
q(u)_{\mathrm{XII}} & =\left((1-u)^{-1 / k}-1\right)^{1 / c},
\end{aligned}
$$

where $u \sim$ iid uniform $(0,1)$ with cdf $u$ and probability density function (pdf) is equal to 1 . The shape of a Burr distribution associated with (3) or (4) is contingent on the values of the shape parameters ( $c$ and $k$ ), which can be determined based on the method of moments (MOM) or the method of percentiles (MOP).

In order to produce a valid Burr Type III or Type XII pdf, the quantile function $q(u)$ in (3) or (4) is required to be a strictly increasing monotone function of $u$ (Headrick et al., 2010). This requirement implies that an inverse function $\left(q^{-1}\right)$ exists such that the cdf associated with $q(u)$ in (3) or (4) can be expressed as $F(q(u))=F(u)=u$. Differentiating both sides of this cdf with respect to $u$ yields the parametric form of pdf for $q(u)$ as $f(q(u))=1 / q^{\prime}(u)$. The simple closed-form expressions for the pdfs associated with (1) and (2) can be given as (Burr, 1942)

$$
\begin{aligned}
& f(x)_{\mathrm{III}}=-c k x^{c-1}\left(1+x^{c}\right)^{-(k+1)}, \\
& f(x)_{\mathrm{XII}}=c k x^{c-1}\left(1+x^{c}\right)^{-(k+1)} .
\end{aligned}
$$

Some of the problems associated with conventional moment-based estimators are that they can be (a) substantially biased, (b) highly dispersed, or (c) influenced by outliers (Headrick, 2011; Hosking, 1990), and thus may not be good representatives of true parameters. To demonstrate, Fig. 1 gives the graph of the pdf associated with the Burr Type III distribution with skew $\left(\gamma_{3}\right)=3$ and kurtosis $\left(\gamma_{4}\right)=65$. Note that the pdf in Fig. 1 has been used in

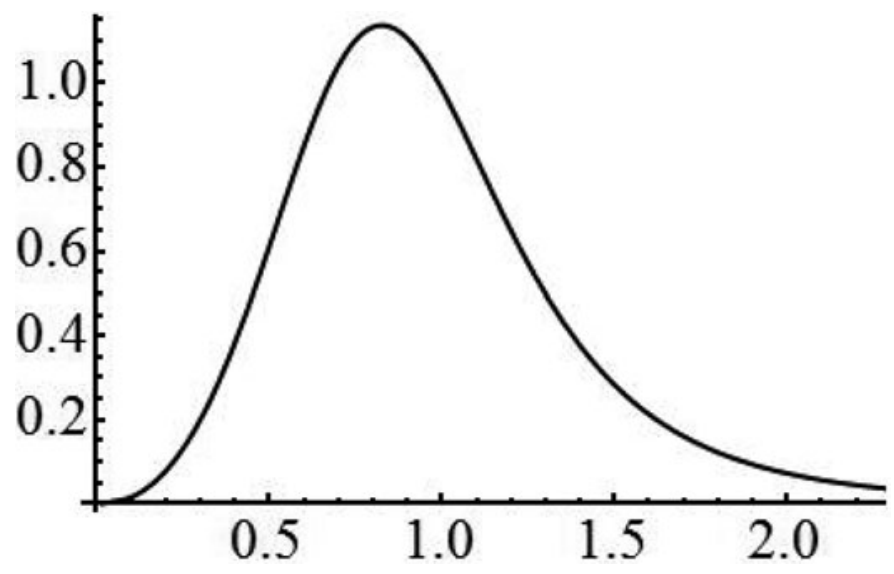

Figure 1. The pdf of the Burr Type III distribution with skew $\left(\gamma_{3}\right)=3$ and kurtosis $\left(\gamma_{4}\right)=65$. The solved values of $c$ and $k$ used in (5) are: $c=-4.406721$ and $k=0.755056$, which are also associated with the values of parameters and their estimates in Table 1. 
Table 1. MOM-based parameter values of skew $\left(\gamma_{3}\right)$ and kurtosis $\left(\gamma_{4}\right)$ and MOP-based parameter values of left-right tail-weight ratio $\left(\xi_{3}\right)$ and tail-weight factor $\left(\xi_{4}\right)$ with their corresponding estimates for the pdf in Fig. 1. Each bootstrapped Estimate, associated $95 \%$ bootstrap $\mathrm{Cl}$, and Std. Error were based on re-sampling 25,000 statistics. Each statistic was based on a sample size of $n=500$.

\begin{tabular}{|c|c|c|c|c|c|}
\hline \multicolumn{3}{|c|}{ Skew: $\gamma_{3}=3$} & \multicolumn{3}{|c|}{ Kurtosis: $\gamma_{4}=65$} \\
\hline Estimate: $\hat{\gamma}_{3}$ & $95 \% \mathrm{Cl}$ & Std. Error & Estimate: $\hat{\gamma}_{4}$ & $95 \% \mathrm{Cl}$ & Std. Error \\
\hline 2.256 & $2.239,2.273$ & 0.00860 & 13.29 & $13.02,13.57$ & 0.14210 \\
\hline \multicolumn{3}{|c|}{ Left-right tail-weight ratio: $\xi_{3}=0.6464$} & \multicolumn{3}{|c|}{ Tail-weight factor: $\xi_{4}=0.4888$} \\
\hline Estimate: $\hat{\xi}_{3}$ & $95 \% \mathrm{Cl}$ & Std. Error & Estimate: $\hat{\xi}_{4}$ & $95 \% \mathrm{Cl}$ & Std. Error \\
\hline 0.6467 & $0.6459,0.6476$ & 0.00041 & 0.4879 & $0.4876,0.4883$ & 0.00017 \\
\hline
\end{tabular}

studies such as Headrick and Pant (2012) and Karian and Dudewicz (2011). Table 1 gives the parameters and sample estimates of skew and kurtosis for the distribution in Fig. 1. Inspection of Table 1 indicates that the bootstrap estimates $\left(\hat{\gamma}_{3}\right.$ and $\left.\hat{\gamma}_{4}\right)$ of skew and kurtosis $\left(\gamma_{3}\right.$ and $\left.\gamma_{4}\right)$ are substantially attenuated below their corresponding parameter values with greater bias and variance as the order of the estimate increases. Specifically, for sample size of $n=500$, the values of the estimates are only $75.2 \%$ and $20.45 \%$ of their corresponding parameters. The estimates $\left(\hat{\gamma}_{3}\right.$ and $\left.\hat{\gamma}_{4}\right)$ of skew and kurtosis $\left(\gamma_{3}\right.$ and $\left.\gamma_{4}\right)$ in Table 1 were calculated based on Fisher's $k$-statistics formulae (see, e.g., Kendall and Stuart, 1977, pp 299-300), currently used by most commercial software packages such as SAS, SPSS, Minitab, etc., for computing the values of skew and kurtosis (where $\gamma_{3,4}=0$ for the standard normal distribution).

The method of percentiles introduced by Karian and Dudewicz (2000) in the context of generalized lambda distributions (GLDs) is an attractive alternative to the method of moments and can be used for estimating shape parameters and fitting distributions to realworld data. The MOP-based GLDs are superior to the MOM-based GLDs for fitting theoretical and empirical distributions that cover a wide range of combinations of skew and kurtosis (Karian and Dudewicz, 2000). Some qualities of the MOP-based procedure in the context of GLDs are that (a) MOP-based procedure can be used to estimate parameters and obtain GLD fits even when the conventional moments associated with a class of GLDs do not exist, (b) the MOP-based procedure for solving equations for the GLD parameters is relatively more accurate than the MOM-based procedure, and (c) the relatively smaller variability of MOP-based sample estimators enables more accurate GLD fits than that achieved through the MOM-based approach (Karian and Dudewicz, 2000). Recently, Kuo and Headrick (2014) have demonstrated that the MOP-based characterization of Tukey's $g$-and- $h$ distributions is superior to the MOM-based characterization in terms of distribution fitting, estimation, relative bias (RB), and relative error. For example, for the Burr Type III pdf in Fig. 1, the MOPbased estimates $\left(\hat{\xi}_{3}\right.$ and $\left.\hat{\xi}_{4}\right)$ of left-right tail-weight ratio and tail-weight factor ( $\xi_{3}$ and $\left.\xi_{4}\right)$ in Table 1 are relatively closer to their respective parameter values with much smaller variance compared with their MOM-based counterparts. Inspection of Table 1 shows that for the sample size of $n=500$, the values of the estimates are on average $100.05 \%$ and $99.82 \%$ of their corresponding parameters.

In view of the above, the main purpose of this study is to characterize the Burr Type III and Type XII distributions through the method of percentiles to obviate the problems associated with MOM-based estimators. Specifically, the purpose of this study is to develop a methodology to simulate the Burr Type III and Type XII distributions with specified values of medians 
$\left(\xi_{1}\right)$, inter-decile ranges $\left(\xi_{2}\right)$, left-right tail-weight ratios (a skew function, $\xi_{3}$ ), and tailweight factors (a kurtosis function, $\xi_{4}$ ). Further, another purpose of this study is to develop a methodology to simulate the correlated Burr Type III and Type XII distributions with a specified Spearman correlation structure.

\section{Methodology}

\subsection{Method of moments-based system}

The MOM-based values of mean $(\mu)$, standard deviation $(\sigma)$, skew $\left(\gamma_{3}\right)$, and kurtosis $\left(\gamma_{4}\right)$ associated with a Burr Type III or Type XII distribution can be given as in Headrick et al. (2010, p. 2211, Eqs. (14)-(17)).

The MOM-based procedure for characterizing Burr Type III and Type XII distributions involves a moment-matching approach in which specified values of skew and kurtosis (obtained from theoretical distributions or real-word data) are substituted on the left-hand sides of Eqs. (16) and (17) from Headrick et al. (2010) for skew $\left(\gamma_{3}\right)$ and kurtosis $\left(\gamma_{4}\right)$, respectively. Subsequently, these equations are simultaneously solved for the shape parameters ( $c$ and $k$ ) associated with Burr Type III and Type XII distributions. The solved values of $c$ and $k$ can be substituted into Eqs. (14) and (15) from Headrick et al. (2010) to determine the values of mean and standard deviation.

\subsection{Method of percentiles-based system}

\subsubsection{General definition}

The MOP-based analogs of location, scale, skew function, and kurtosis function are respectively defined by median $\left(\xi_{1}\right)$, inter-decile range $\left(\xi_{2}\right)$, left-right tail-weight ratio $\left(\xi_{3}\right)$, and tail-weight factor $\left(\xi_{4}\right)$, and expressed as (Karian and Dudewicz, 2000, pp. 154-155)

$$
\begin{aligned}
& \xi_{1}=\pi_{p=0.50}, \\
& \xi_{2}=\pi_{p=0.90}-\pi_{p=0.10}, \\
& \xi_{3}=\frac{\pi_{p=0.50}-\pi_{p=0.10}}{\pi_{p=0.90}-\pi_{p=0.50}}, \\
& \xi_{4}=\frac{\pi_{p=0.75}-\pi_{p=0.25}}{\xi_{2}},
\end{aligned}
$$

where $\pi_{p}$ in $(7)-(10)$ is the $(100 p)$ th percentile and where $p \in(0,1)$. Note that the quantile function $q(u)$ in (3) or (4) can be considered as a substitute of $\pi_{p}$ in (7)-(10) in the context of Burr Type III and Type XII distributions as $u \in(0,1)$.

\subsubsection{MOP-based Burr Type III distribution}

Substituting $q(u)$ from (3) into (7)-(10) and simplifying the resulting expressions, the MOPbased system of Burr Type III distribution is given as follows:

$$
\begin{aligned}
& \xi_{1}=\left(2^{1 / k}-1\right)^{1 / c}, \\
& \xi_{2}=\left((10 / 9)^{1 / k}-1\right)^{1 / c}-\left(10^{1 / k}-1\right)^{1 / c},
\end{aligned}
$$




$$
\begin{aligned}
& \xi_{3}=\frac{\left(2^{1 / k}-1\right)^{1 / c}-\left(10^{1 / k}-1\right)^{1 / c}}{\left((10 / 9)^{1 / k}-1\right)^{1 / c}-\left(2^{1 / k}-1\right)^{1 / c}}, \\
& \xi_{4}=\frac{\left((4 / 3)^{1 / k}-1\right)^{1 / c}-\left(4^{1 / k}-1\right)^{1 / c}}{\left((10 / 9)^{1 / k}-1\right)^{1 / c}-\left(10^{1 / k}-1\right)^{1 / c}} .
\end{aligned}
$$

\subsubsection{MOP-based Burr Type XII distribution}

Substituting $q(u)$ from (4) into (7)-(10) and simplifying the resulting expressions, the MOPbased system of Burr Type XII distributions is given as follows:

$$
\begin{aligned}
& \xi_{1}=\left(2^{1 / k}-1\right)^{1 / c}, \\
& \xi_{2}=\left(10^{1 / k}-1\right)^{1 / c}-\left((10 / 9)^{1 / k}-1\right)^{1 / c}, \\
& \xi_{3}=\frac{\left(2^{1 / k}-1\right)^{1 / c}-\left((10 / 9)^{1 / k}-1\right)^{1 / c}}{\left(10^{1 / k}-1\right)^{1 / c}-\left(2^{1 / k}-1\right)^{1 / c}}, \\
& \xi_{4}=\frac{\left(4^{1 / k}-1\right)^{1 / c}-\left((4 / 3)^{1 / k}-1\right)^{1 / c}}{\left(10^{1 / k}-1\right)^{1 / c}-\left((10 / 9)^{1 / k}-1\right)^{1 / c}} .
\end{aligned}
$$

In the context of Burr Type III and Type XII distributions, the parameter values of median $\left(\xi_{1}\right)$, inter-decile range $\left(\xi_{2}\right)$, left-right tail-weight ratio $\left(\xi_{3}\right)$, and tail-weight factor $\left(\xi_{4}\right)$ in (11)-(14) or in (15)-(18) have the following restrictions:

$$
0<\xi_{1}<+\infty, \quad \xi_{2} \geq 0, \quad \xi_{3} \geq 0, \quad 0 \leq \xi_{4} \leq 1,
$$

where a symmetric distribution will have $\xi_{1}=$ median $=$ mean and $\xi_{3}=1$.

For the specified values of left-right tail-weight ratio $\left(\xi_{3}\right)$ and tail-weight factor $\left(\xi_{4}\right)$, the systems of Eqs. (13)-(14) and (17)-(18) can be simultaneously solved for real values of $c$ and $k$. The solved values of $c$ and $k$ can be substituted in (3) and (4), respectively, for generating the Burr Type III and Type XII distributions. Further, the solved values of $c$ and $k$ can be substituted in (11)-(12) and (15)-(16) to determine the values of median $\left(\xi_{1}\right)$ and interdecile range $\left(\xi_{2}\right)$ associated with the Type III and Type XII distributions, respectively.

Let $X_{1}<X_{2}<X_{3}<\cdots<X_{i}<X_{i+1}<\cdots<X_{n}$ be the order statistics of a sample $\left(Y_{1}, Y_{2}, Y_{3}, \ldots, Y_{n}\right)$ of size $n$. Let $\hat{\pi}_{p}$ be the $(100 p)$ th percentile from this sample, where $p \in(0,1)$. Let $(n+1) p=i+(a / b)$, where $i$ is the positive integer and $a / b$ is the proper fraction. Then $\hat{\pi}_{p}$ can be computed as (Karian and Dudewicz, 2000, p. 154)

$$
\hat{\pi}_{p}=X_{i}+(a / b)\left(X_{i+1}-X_{i}\right)
$$

For a sample of size $n$, the MOP-based estimates of $\xi_{1}-\xi_{4}$ in (11)-(14) or (15)-(18) can be computed in two steps as follows: (a) compute the values of 10th, 25th, 50th, 75th, and 90th percentiles using (20), and (b) substitute these percentiles into (7)-(10) to obtain the sample estimates of $\xi_{1}-\xi_{4}$. In the next section, examples are provided to demonstrate the aforementioned methodology and the advantages of MOP procedure over the conventional MOM procedure in the contexts of distribution fitting and estimation. 


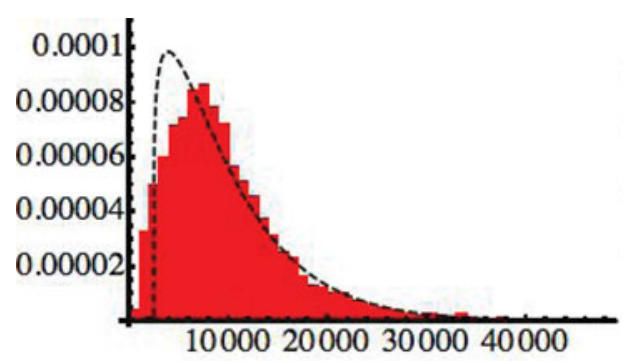

MOM-based Burr Type XII pdf superimposed on the histogram of total hospital charges data of 12,145 heart attack patients $(n=12,145)$.

\begin{tabular}{ll}
\hline Estimates & Shape parameters \\
\hline $\bar{X}=9879.1$ & $c=1.166499$ \\
$S=6558.4$ & $k=40.985551$ \\
$\hat{\gamma}_{3}=1.703547$ & \\
$\hat{\gamma}_{4}=4.339990$ &
\end{tabular}

(A)

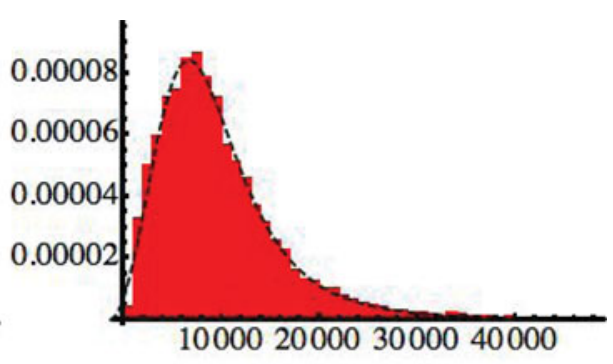

MOP-based Burr Type XII pdf superimposed on the histogram of total hospital charges data of 12,145 heart attack patients $(n=12,145)$.

\begin{tabular}{ll}
\hline Estimates & Shape parameters \\
\hline$m_{1}=8445$ & $c=2.779328$ \\
$m_{2}=14684.8$ & $k=1.383305$ \\
$\xi_{3}=0.547691$ & \\
$\hat{\xi}_{4}=0.486762$ &
\end{tabular}

(B)

Figure 2. Histograms of the total hospital charges (in US dollars) data of 12,145 heart attack patients superimposed by the (a) MOM- and (b) MOP-based Burr Type XII pdfs.

\section{Comparison of MOP-based procedure with MOM-based procedure}

\subsection{Distribution fitting}

Figure 2 shows the conventional MOM- and MOP-based Burr Type XII pdfs superimposed on the histogram of total hospital charges (in US dollars) data of 12,145 heart attack patients discharged from all of the hospitals in New York State in 1993. There were 12,844 cases with 699 missing values for the total hospital charges. See the website: http://wiki.stat.ucla.edu/socr/index.php/SOCR_Data_AMI_NY_1993_HeartAttacks

The conventional MOM-based estimates $\left(\hat{\gamma}_{3}\right.$ and $\left.\hat{\gamma}_{4}\right)$ of skew and kurtosis $\left(\gamma_{3}\right.$ and $\left.\gamma_{4}\right)$ and the MOP-based estimates $\left(\hat{\xi}_{3}\right.$ and $\left.\hat{\xi}_{4}\right)$ of left-right tail-weight ratio and tail-weight factor $\left(\xi_{3}\right.$ and $\left.\xi_{4}\right)$ were computed for the sample of size $n=12,145$ patients. The estimates of $\gamma_{3}$ and $\gamma_{4}$ were computed based on Fisher's $k$-statistics formulae (Kendall and Stuart, 1977, pp. 47-48), whereas the estimates of $\xi_{3}$ and $\xi_{4}$ were computed using (7)-(10) and (20), respectively. These sample estimates were then used to solve the values of shape parameters ( $c$ and $k$ ) using (a) Eqs. (16) and (17) from Headrick et al. (2010), and (b) Eqs. (17) and (18), respectively, for the MOM- and MOP-based fits. The solved values of $c$ and $k$ were subsequently used in (6) to superimpose the Burr Type XII pdfs shown in Fig. 2.

To superimpose the Burr Type XII pdf (dashed curves), the quantile function $q(u)$ from (4) was transformed as (a) $\bar{X}+S(q(u)-\mu) / \sigma$, and (b) $m_{1}+m_{2}\left(q(u)-\xi_{1}\right) / \xi_{2}$, respectively, where $(\bar{X}, S)$ and $(\mu, \sigma)$ are the values of (mean, standard deviation), and $\left(m_{1}, m_{2}\right)$ and $\left(\xi_{1}, \xi_{2}\right)$ are the values of (median, inter-decile range) obtained from the actual data and the Burr Type XII pdf, respectively.

Inspection of the two panels in Fig. 2 illustrates that the MOP-based Burr Type XII pdf provides a better fit to the total hospital charges data. The Chi-square goodness of fit statistics along with their corresponding $p$-values in Table 2 provide evidence that the MOM-based 
Table 2. Chi-square goodness of fit statistics for the conventional MOM- and MOP-based Burr Type XII approximations for the total hospital charges $(n=12,145)$ data in Fig. 2 .

\begin{tabular}{cccccc}
\hline$\%$ & Expected & $\begin{array}{c}\text { Obs. } \\
\text { (MOM) }\end{array}$ & $\begin{array}{c}\text { Obs. } \\
(\text { MOP) }\end{array}$ & $\begin{array}{c}\text { Total hospital } \\
\text { charges (MOM) }\end{array}$ & $\begin{array}{c}\text { Total hospital } \\
\text { charges (MOP) }\end{array}$ \\
\hline 10 & 1214.5 & 1444 & 1214 & $<3537.98$ & $<3248.40$ \\
20 & 1214.5 & 809 & 1214 & $3537.98-4554.41$ & $3248.40-4763.57$ \\
30 & 1214.5 & 968 & 1147 & $4554.41-5617.68$ & $4763.57-6015.52$ \\
40 & 1214.5 & 1127 & 1236 & $5617.68-6779.64$ & $6015.52-7207.91$ \\
50 & 1214.5 & 1372 & 1262 & $6779.64-8095.82$ & $7207.91-8445.00$ \\
60 & 1214.5 & 1452 & 1243 & $8095.82-9648.28$ & $8445.00-9827.63$ \\
70 & 1214.5 & 1344 & 1164 & $9648.28-11583.63$ & $9827.63-11510.03$ \\
80 & 1214.5 & 1353 & 1225 & $11583.63-14224.23$ & $11510.03-13828.69$ \\
90 & 1214.5 & 1175 & 1226 & $14224.23-18585.93$ & $13828.69-17933.20$ \\
100 & 1214.5 & 1101 & 1214 & 18585.93 or more & 17933.20 or more \\
& & & & $\chi^{2}=343.46$ & $\chi^{2}=8.96$ \\
& & & & $p<0.0001$ & $p<0.1107$ \\
\hline
\end{tabular}

Burr Type XII pdf does not provide a good fit to these real-world data, whereas the MOPbased Burr Type XII pdf fits very well. The degrees of freedom for the Chi-square goodness of fit tests were computed as $d f=5=10$ (class intervals) - 4 (estimates of the parameters) 1 (sample size).

\subsection{Estimation}

An example to demonstrate the advantages of MOP-based estimation over the conventional MOM-based estimation is provided in Fig. 3 and Tables 3-6. Given in Fig. 3 are the pdfs of the $F(3,10)$, Chi-square $(d f=1)$, extreme value $(0,1)$, and logistic $(0,1)$ distributions superimposed, respectively, by the Burr Type XII, Type III, Type XII, and Type III pdfs (dashed curves) in both (a) conventional MOM-, and (b) MOP-based systems. The conventional MOM-based parameters of skew $\left(\gamma_{3}\right)$ and kurtosis $\left(\gamma_{4}\right)$ associated with these four distributions, given in Table 3, were computed by using Eqs. (11)-(13) from Headrick et al. (2010, p. 2211). The values of shape parameters ( $c$ and $k$ ) given in Table 3 were determined by simultaneously solving

Table 3. MOM-based parameters of the mean $(\mu)$, standard deviation $(\sigma)$, skew $\left(\gamma_{3}\right)$, and kurtosis $\left(\gamma_{4}\right)$ along with the values of shape parameters ( $c$ and $k$ ) for the four distributions (dashed curves) in Fig. 3 (panel A).

\begin{tabular}{lcccccc}
\hline Distribution & $\mu$ & $\sigma$ & $\gamma_{3}$ & $\gamma_{4}$ & $c$ & $k$ \\
\hline 1 & 0.306182 & 0.362801 & 4.221159 & 59.454545 & 1.097740 & 4.481719 \\
2 & 0.139427 & 0.263634 & 2.828427 & 12.0 & -5.542062 & 0.027237 \\
3 & 0.415523 & 0.242125 & 1.139547 & 2.4 & 2.025127 & 5.374401 \\
4 & 0.937510 & 0.139248 & 0.0 & 1.2 & -15.776368 & 0.540152 \\
\hline
\end{tabular}

Table 4. MOP-based parameters of median $\left(\xi_{1}\right)$, inter-decile range $\left(\xi_{2}\right)$, left-right tail-weight ratio $\left(\xi_{3}\right)$, and tail-weight factor $\left(\xi_{4}\right)$ along with the values of shape parameters ( $c$ and $k$ ) for the four distributions (dashed curves) in Fig. 3 (panel B).

\begin{tabular}{lcccccc}
\hline Distribution & $\xi_{1}$ & $\xi_{2}$ & $\xi_{3}$ & $\xi_{4}$ & $c$ & $k$ \\
\hline 1 & 0.326084 & 1.008596 & 0.347335 & 0.470650 & 1.240409 & 3.116624 \\
2 & 0.198340 & 1.187837 & 0.195123 & 0.454232 & -2.113101 & 0.200868 \\
3 & 0.615990 & 0.883690 & 0.637281 & 0.509835 & 2.446790 & 2.599457 \\
4 & 0.907056 & 0.508122 & 1.0 & 0.5 & -10.148568 & 0.530756 \\
\hline
\end{tabular}



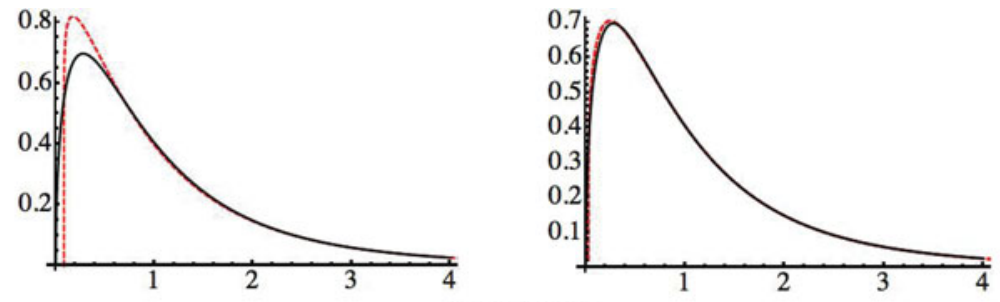

Distribution 1
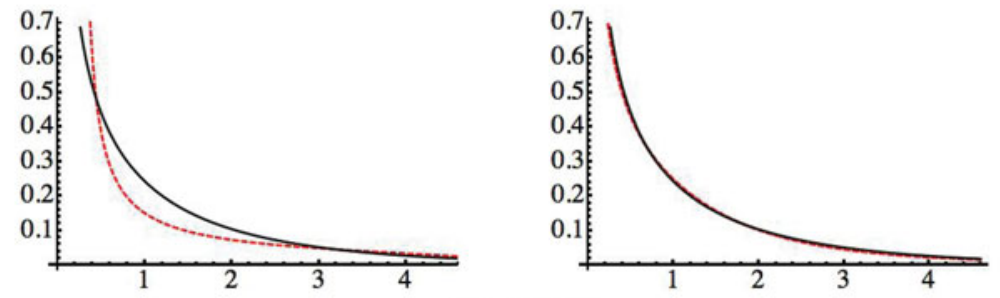

Distribution 2
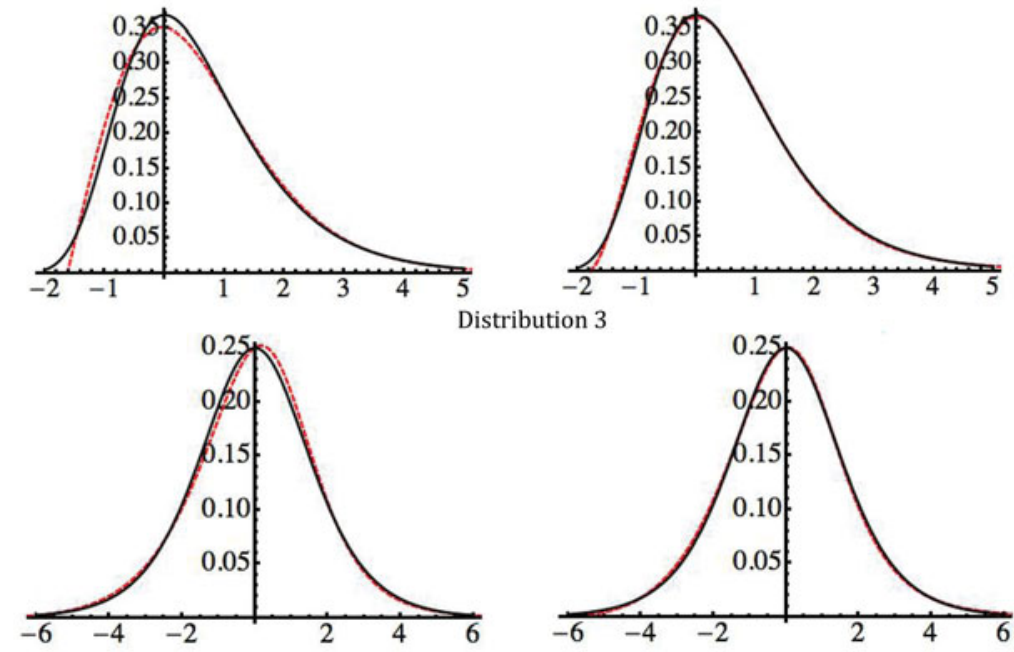

(A)

(B)

Figure 3. The pdfs (dashed curves) of the four distributions: Distribution 1. Burr Type XII $\approx F(3,10)$, Distribution 2. Burr Type III $\approx$ Chi-square $(d f=1)$, Distribution 3 . Burr Type XII $\approx$ extreme value $(0,1)$, and Distribution 4. Burr Type III $\approx \operatorname{logistic}(0,1)$ superimposed by (a) MOM-, and (b) MOP-based Burr Type III and Type XII pdfs, respectively.

Eqs. (16) and (17) from Headrick et al. (2010, p. 2211). The values of $c$ and $k$ were used in (5) and (6) to superimpose the conventional MOM-based Burr Type XII, Type III, Type XII, and Type III distributions, respectively, as shown in Fig. 3 (panel A).

The MOP-based parameters of left-right tail-weight ratio $\left(\xi_{3}\right)$ and tail-weight factor $\left(\xi_{4}\right)$ associated with the four distributions in Fig. 3, given in Table 4, were obtained in two steps as follows: (a) Compute the values of 10th, 25th, 50th, 75th, and 90th percentiles from the cdfs of the four distributions, and (b) substitute these five percentiles into (7)-(10) to compute the values of $\xi_{3}$ and $\xi_{4}$. The values of shape parameters ( $c$ and $k$ ) given in Table 4 were determined by solving the systems of Eqs. (13)-(14) and (17)-(18), respectively. These values of $c$ and $k$ were used in (5) and (6) to superimpose the MOP-based Burr Type XII, Type III, Type XII, and Type III distributions, respectively, as shown in Fig. 3 (panel B). 
Table 5. Skew $\left(\gamma_{3}\right)$ and Kurtosis $\left(\gamma_{4}\right)$ results for the conventional MOM-based procedure.

\begin{tabular}{|c|c|c|c|c|c|}
\hline Distribution & Parameter & Estimate & $95 \%$ Bootstrap Cl & Std. Error & $R B \%$ \\
\hline \multicolumn{6}{|c|}{$n=25$} \\
\hline \multirow[t]{2}{*}{1} & $\gamma_{3}=4.2212$ & $\hat{\gamma}_{3}=1.75$ & $1.7397,1.7597$ & 0.00510 & -58.54 \\
\hline & $\gamma_{4}=59.4545$ & $\hat{\gamma}_{4}=3.34$ & $3.2911,3.3871$ & 0.02430 & -94.38 \\
\hline \multirow[t]{2}{*}{2} & $\gamma_{3}=2.8284$ & $\hat{\gamma}_{3}^{4}=2.151$ & $2.1428,2.1596$ & 0.00429 & -23.95 \\
\hline & $\gamma_{4}=12.0$ & $\hat{\gamma}_{4}^{3}=4.215$ & $4.1715,4.2614$ & 0.02306 & -64.68 \\
\hline \multirow[t]{2}{*}{3} & $\gamma_{3}=1.1395$ & $\hat{\gamma}_{3}=0.7929$ & $0.7859,0.7994$ & 0.00346 & -30.42 \\
\hline & $\gamma_{4}=2.4$ & $\hat{\gamma}_{4}=0.5014$ & $0.4786,0.5220$ & 0.01111 & -79.11 \\
\hline \multirow[t]{2}{*}{4} & $\gamma_{3}^{4}=0.0$ & $\hat{\gamma}_{3}=-0.051$ & $-0.0585,-0.0430$ & 0.00391 & - \\
\hline & $\gamma_{4}=1.2$ & $\begin{array}{r}\hat{\gamma}_{4}=0.3396 \\
n=\end{array}$ & $0.3243,0.3552$ & 0.00791 & -71.70 \\
\hline 1 & $\gamma_{3}=4.2212$ & $\hat{\gamma}_{3}=3.606$ & $3.5888,3.6275$ & 0.00972 & -14.57 \\
\hline & $\gamma_{4}=59.4545$ & $\hat{\gamma}_{4}=26.29$ & $25.8657,26.7366$ & 0.22370 & -55.78 \\
\hline \multirow[t]{2}{*}{2} & $\gamma_{3}=2.8284$ & $\hat{\gamma}_{3}=2.761$ & $2.7548,2.7681$ & 0.00335 & -2.38 \\
\hline & $\gamma_{4}=12.0$ & $\hat{\gamma}_{4}^{3}=10.17$ & $10.0615,10.3107$ & 0.06265 & -15.25 \\
\hline \multirow[t]{2}{*}{3} & $\gamma_{3}=1.1395$ & $\hat{\gamma}_{3}=1.124$ & $1.1219,1.1266$ & 0.00122 & -1.36 \\
\hline & $\gamma_{4}=2.4$ & $\hat{\hat{\gamma}}_{4}^{3}=2.266$ & $2.2451,2.2861$ & 0.01048 & -5.58 \\
\hline \multirow[t]{2}{*}{4} & $\gamma_{3}^{4}=0.0$ & $\hat{\gamma}_{3}=-0.0045$ & $-0.0064,-0.0025$ & 0.00101 & - \\
\hline & $\gamma_{4}=1.2$ & $\hat{\gamma}_{4}=1.158$ & $1.1487,1.1683$ & 0.00498 & -3.5 \\
\hline
\end{tabular}

To superimpose Burr Type III or Type XII distribution, the quantile function $q(u)$ in (3) or (4) was transformed into: (a) $\bar{X}+S(q(u)-\mu) / \sigma$, and (b) $m_{1}+m_{2}\left(q(u)-\xi_{1}\right) / \xi_{2}$, respectively, where $(\bar{X}, S)$ and $(\mu, \sigma)$ are the values of (mean, standard deviation), and $\left(m_{1}, m_{2}\right)$ and $\left(\xi_{1}, \xi_{2}\right)$ are the values of (median, inter-decile range) obtained from the original distribution and the respective Burr Type III or Type XII approximation, respectively.

The advantages of MOP-based estimators over the MOM-based estimators can also be demonstrated in the context of Burr Type III and Type XII distributions by considering the Monte Carlo simulation results associated with the indices for the percentage of relative bias (RB\%) and standard error (Std. Error) reported in Tables 5 and 6.

Table 6. Left-right tail-weight ratio $\left(\xi_{3}\right)$ and tail-weight factor $\left(\xi_{4}\right)$ results for the MOP-based procedure.

\begin{tabular}{|c|c|c|c|c|c|}
\hline Distribution & Parameter & Estimate & 95\% Bootstrap Cl & Std. Error & $\mathrm{RB} \%$ \\
\hline \multicolumn{6}{|c|}{$\mathrm{n}=25$} \\
\hline \multirow[t]{2}{*}{1} & $\xi_{3}=0.347335$ & $\hat{\xi}_{3}=0.3646$ & $0.3625,0.3671$ & 0.00120 & 4.98 \\
\hline & $\xi_{4}=0.470650$ & $\hat{\xi_{4}}=0.4261$ & $0.4545,0.4277$ & 0.00080 & -9.46 \\
\hline \multirow[t]{2}{*}{2} & $\xi_{3}=0.195123$ & $\hat{\xi_{3}}=0.2161$ & $0.2143,0.2178$ & 0.00089 & 10.76 \\
\hline & $\xi_{4}=0.454232$ & $\hat{\xi}_{4}=0.4026$ & $0.4008,0.4044$ & 0.00090 & -11.36 \\
\hline \multirow[t]{2}{*}{3} & $\xi_{3}=0.637281$ & $\hat{\xi}_{3}=0.6887$ & $0.6850,06928$ & 0.00197 & 8.07 \\
\hline & $\xi_{4}=0.509835$ & $\hat{\xi}_{4}=0.4702$ & $0.4688,0.4715$ & 0.00066 & -7.77 \\
\hline \multirow[t]{2}{*}{4} & $\xi_{3}=1.0$ & $\hat{\xi}_{3}=1.0960$ & $1.0901,1.1024$ & 0.00313 & 9.6 \\
\hline & $\xi_{4}=0.5$ & $\begin{array}{r}\hat{\xi}_{4}=0.4623 \\
n=\end{array}$ & $0.4610,0.4635$ & 0.00063 & -7.54 \\
\hline \multirow[t]{2}{*}{1} & $\xi_{3}=0.347335$ & $\hat{\xi}_{3}=0.3481$ & $0.3478,0.3485$ & 0.00017 & 0.23 \\
\hline & $\xi_{4}=0.470650$ & $\hat{\xi}_{4}=0.4703$ & $0.4700,0.4705$ & 0.00014 & -0.06 \\
\hline \multirow[t]{2}{*}{2} & $\xi_{3}=0.195123$ & $\xi_{3}=0.1961$ & $0.1959,0.1963$ & 0.00012 & 0.51 \\
\hline & $\xi_{4}=0.454232$ & $\hat{\xi_{4}}=0.4541$ & $0.4538,0.4544$ & 0.00016 & - \\
\hline \multirow[t]{2}{*}{3} & $\xi_{3}=0.637281$ & $\hat{\xi}_{3}=0.6379$ & $0.6373,0.6384$ & 0.00030 & - \\
\hline & $\xi_{4}=0.509835$ & $\hat{\xi_{4}}=0.5094$ & $0.5092,0.5096$ & 0.00012 & -0.08 \\
\hline \multirow[t]{2}{*}{4} & $\xi_{3}=1.0$ & $\hat{\xi}_{3}=1.001$ & $0.9997,1.0015$ & 0.00044 & - \\
\hline & $\xi_{4}=0.5$ & $\hat{\xi}_{4}=0.4996$ & $0.4993,0.4998$ & 0.00011 & -0.84 \\
\hline
\end{tabular}


Specifically, a Fortran (Microsoft Corporation, 1994) algorithm was written to simulate 25,000 independent samples of sizes $n=25$ and 1000, and the conventional MOM-based estimates $\left(\hat{\gamma}_{3}\right.$ and $\left.\hat{\gamma}_{4}\right)$ of skew and kurtosis $\left(\gamma_{3}\right.$ and $\left.\gamma_{4}\right)$ and the MOP-based estimates $\left(\hat{\xi}_{3}\right.$ and $\left.\hat{\xi}_{4}\right)$ of left-right tail-weight ratio and tail-weight factor $\left(\xi_{3}\right.$ and $\xi_{4}$ ) were computed for each of the $(2 \times 25,000)$ samples based on the parameters and the values of $c$ and $k$ listed in Tables 3 and 4 . The estimates $\left(\hat{\gamma}_{3}\right.$ and $\left.\hat{\gamma}_{4}\right)$ of $\gamma_{3}$ and $\gamma_{4}$ were computed based on Fisher's $k$-statistics formulae (Kendall and Stuart, 1977, pp. 47-48), whereas the estimates $\left(\hat{\xi}_{3}\right.$ and $\left.\hat{\xi}_{4}\right)$ of $\xi_{3}$ and $\xi_{4}$ were computed using (7)-(10) and (20). Bias-corrected accelerated bootstrapped average estimates (Estimate), associated $95 \%$ confidence intervals ( $95 \%$ bootstrap CI), and Std. Errors were obtained for each type of estimate using 10,000 re-samples via the commercial software package Spotfire S+ (TIBCO, 2010). Further, if a parameter was outside its associated 95\% bootstrap $\mathrm{CI}$, then the RB\% was computed for the estimate as follows:

$$
\mathrm{RB} \%=100 \times(\text { Estimate }- \text { Parameter }) / \text { Parameter } .
$$

The results in Tables 5 and 6 illustrate that the MOP-based estimators are superior to their conventional MOM-based counterparts in terms of both smaller relative bias and error. These advantages are most pronounced in the context of smaller sample sizes and higher order moments. For example, for Distribution 1, given a sample of size $n=25$, the conventional moment-based estimators $\left(\hat{\gamma}_{3}\right.$ and $\left.\hat{\gamma}_{4}\right)$ generated in the simulation were, on average, $41.46 \%$ and $5.62 \%$ of their corresponding parameters $\left(\gamma_{3}\right.$ and $\left.\gamma_{4}\right)$. On the other hand, for the same Distribution 1, the MOP-based estimators $\left(\hat{\xi}_{3}\right.$ and $\left.\hat{\xi}_{4}\right)$ generated in the simulation were, on average, $104.98 \%$ and $90.52 \%$ of their corresponding parameters $\left(\xi_{3}\right.$ and $\left.\xi_{4}\right)$. Thus, the relative biases of MOP-based estimators are essentially negligible compared with those associated with the MOM-based estimators. Also, it can be verified that the standard errors associated with estimators $\hat{\xi}_{3}$ and $\hat{\xi}_{4}$ are relatively much smaller and more stable than the standard errors associated with estimators $\hat{\gamma}_{3}$ and $\hat{\gamma}_{4}$. Inspection of the graphs in Fig. 3 (panels A and B) and the Monte Carlo simulation results in Tables 5 and 6 illustrate that the MOP-based Burr Type III and Type XII approximations provide more accurate fits to the four distributions than the MOM-based approximations.

\section{Spearman correlation for the Burr Type III and Type XII distributions}

Let $Y_{j}=q\left(u_{j}\right)$ and $Y_{k}=q\left(u_{k}\right)$ be two random Burr Type III or Type XII variables as defined in (3) or (4) with cdfs denoted as $F\left(Y_{j}\right)=u_{j}$ and $F\left(Y_{k}\right)=u_{k}$, respectively. Let $Y_{j}=q\left(u_{j}\right)$ and $Y_{k}=q\left(u_{k}\right)$ produce valid Burr Type III or Type XII pdfs and be strictly increasing monotonic transformations of $u_{j}$ and $u_{k}$, which are strictly increasing monotonic transformations of standard normal random variates $Z_{j}$ and $Z_{k}$. Thus, the rank orders $R\left(Y_{j}\right)$ and $R\left(Z_{j}\right)$ of $Y_{j}$ and $Z_{j}$ are identical, and so are the rank orders $R\left(Y_{k}\right)$ and $R\left(Z_{k}\right)$ of $Y_{k}$ and $Z_{k}$. This implies that Spearman correlations $\eta_{R\left(Y_{j}\right), R\left(Z_{j}\right)}=\eta_{R\left(Y_{k}\right), R\left(Z_{k}\right)}=1$.

Suppose we would like to simulate a $T$-variate Burr Type III and Type XII distributions from the quantile functions in (3) and (4) with a specified $T \times T$ Spearman correlation matrix and where each distribution has specified values of left-right tail-weight ratio $\left(\xi_{3}\right)$ and tailweight factor $\left(\xi_{4}\right)$. Specifically, let $Z_{1}, \ldots, Z_{T}$ denote standard normal variables with cdfs and the joint pdf associated with $Z_{j}$ and $Z_{k}$ given by the following expressions:

$$
\Phi\left(Z_{j}\right)=\int_{-\infty}^{z_{j}}(2 \pi)^{-1 / 2} \exp \left\{-v_{j}^{2} / 2\right\} d v_{j},
$$




$$
\begin{aligned}
\Phi\left(Z_{k}\right) & =\int_{-\infty}^{z_{k}}(2 \pi)^{-1 / 2} \exp \left\{-v_{k}^{2} / 2\right\} d v_{k} \\
f_{j k} & =\left(2 \pi\left(1-r_{z_{j}, z_{k}}^{2}\right)^{1 / 2}\right)^{-1} \exp \left\{-\left(2\left(1-r_{z_{j}, z_{k}}^{2}\right)\right)^{-1}\left(z_{j}^{2}+z_{k}^{2}-2 r_{z_{j}, z_{k}} z_{j} z_{k}\right)\right\}
\end{aligned}
$$

where $r_{z_{j}, z_{k}}$ in (24) is the intermediate correlation (IC) between $Z_{j}$ and $Z_{k}$. Using the cdfs in (22) and (23) as zero-one uniform deviates, i.e., $u_{j}=\Phi\left(Z_{j}\right), u_{k}=\Phi\left(Z_{k}\right) \sim U(0,1)$, the quantile function defined in either (3) or (4) can be expressed as a function of $\Phi\left(Z_{j}\right)$, or $\Phi\left(Z_{k}\right) \quad$ (e.g., $q_{j}\left(\Phi\left(Z_{j}\right)\right)$ or $\left.q_{k}\left(\Phi\left(Z_{k}\right)\right)\right)$. Also, since the quantile functions in (3) and (4) are strictly increasing monotonic transformations, the rank correlations $\eta_{R\left(Y_{j}\right), R\left(Y_{k}\right)}$ and $\eta_{R\left(Z_{j}\right), R\left(Z_{k}\right)}$ are equal. Thus, the Spearman correlation of $Y_{j}=q_{j}\left(\Phi\left(Z_{j}\right)\right)$ and $Y_{k}=q_{k}\left(\Phi\left(Z_{k}\right)\right)$ can be determined (see Eq. (4.3) in Headrick, 2010, or Eq. (2) in (Moran, 1948) as

$$
\eta_{j k}=\eta_{R\left(Y_{j}\right), R\left(Y_{k}\right)}=\left(\frac{6}{\pi}\right)\left\{\left(\frac{n-2}{n+1}\right) \sin ^{-1}\left(\frac{1}{2} r_{z_{j}, z_{k}}\right)+\left(\frac{1}{n+1}\right) \sin ^{-1}\left(r_{z_{j}, z_{k}}\right)\right\},
$$

where $\eta_{j k}=\eta_{R\left(Y_{j}\right), R\left(Y_{k}\right)}$ is the specified Spearman correlation between $Y_{j}$ and $Y_{k}$ and $r_{z_{j}, z_{k}}$ is the Pearson intermediate correlation between $Z_{j}$ and $Z_{k}$. For a specified value of $\eta_{j k}=$ $\eta_{R\left(Y_{j}\right), R\left(Y_{k}\right)}$ and a given sample size $(n)$, the value of intermediate correlation $\left(r_{z_{j}, z_{k}}\right)$ can be computed by numerically solving (25).

Provided in Algorithm 1 is a source code written in Mathematica (Wolfram, 2003; Wolfram Research, 2011), which shows an example for computing intermediate correlation $\left(r_{z_{j}, z_{k}}\right)$ for the Spearman correlation procedure. The steps for simulating the correlated Burr Type III and Type XII distributions with specified values of left-right tail-weight ratio $\left(\xi_{3}\right)$ and tail-weight factor $\left(\xi_{4}\right)$, and with specified Spearman correlation structure are given in Section 5.

Algorithm 1. Mathematica source code for computing intermediate correlations for specified Spearman correlation procedure. The example is for Distribution $j=1$ and Distribution $k=$ $2\left(\eta_{12}\right)$. See dashed curves of Distribution 1 and Distribution 2 in Figure 3 (Panel B) and specified correlation in Table 7.

(* Sample size *)

$n=25$;

( ${ }^{*}$ Intermediate correlation *)

$r_{z_{1}, z_{2}}=0.834779$;

(* Compute the specified Spearman correlation using equation (4.34) from Headrick [28] *)

$\eta_{12}=\left(\frac{6}{\pi}\right)\left\{\left(\frac{n-2}{n+1}\right) \sin ^{-1}\left(\frac{1}{2} r_{z_{1}, z_{2}}\right)+\left(\frac{1}{n+1}\right) \sin ^{-1}\left(r_{z_{1}, z_{2}}\right)\right\}$

0.80

\section{The procedure for Monte Carlo simulation with an example}

The procedure for simulating Burr Type III and Type XII distributions with specified values of $\xi_{3}, \xi_{4}$, and Spearman correlations can be summarized in the following six steps: 
Table 7. Specified correlations for the distributions in Fig. 3 (panels A and B).

\begin{tabular}{lllll}
\hline Distribution & 1 & 2 & 3 & 4 \\
\hline 1 & 1.0 & & & \\
2 & 0.80 & 1.0 & 1.0 & 1.0 \\
3 & 0.75 & 0.70 & 0.40 & 1.0 \\
4 & 0.60 & 0.50 & . & \\
\hline
\end{tabular}

1. Specify the values of $\xi_{3}$ and $\xi_{4}$ for the $T$ of the form in (3) and (4), i.e., $q_{1}\left(\Phi\left(z_{1}\right)\right), \ldots, q_{T}\left(\Phi\left(z_{T}\right)\right)$ and obtain the solutions for the shape parameters $\mathrm{c}$ and $k$ by simultaneously solving the systems of Eqs. (13)-(14) and (17)-(18) using the specified values of $\xi_{3}$ and $\xi_{4}$ for each distribution. Specify the $T \times T$ matrix of Spearman correlations $\left(\eta_{j k}\right)$ between $q_{j}\left(\Phi\left(z_{j}\right)\right)$ and $q_{k}\left(\Phi\left(Z_{k}\right)\right)$, where $j<k \in$ $\{1,2, \ldots, T\}$.

2. Compute the values of intermediate (Pearson) correlation, $r_{z_{j}, z_{k}}$, by substituting the value of specified Spearman correlation $\left(\eta_{j k}\right)$ and the sample size $(n)$ into the leftand the right-hand sides of (25), respectively, and then numerically solving (25) for $r_{z_{j}, z_{k}}$. See Algorithm 1 for an example. Repeat this step separately for all $T(T-1) / 2$ pair-wise combinations of intermediate correlations.

3. Assemble the intermediate correlations computed in Step 2 into the $T \times T$ matrix and decompose this matrix using the Cholesky factorization. Note that this step requires the intermediate correlation matrix to be positive definite.

4. Use elements of the matrix resulting from the Cholesky factorization of Step 3 to generate $T$ standard normal variates $\left(Z_{1}, \ldots, Z_{T}\right)$ correlated at the intermediate correlation levels as follows:

$$
\begin{aligned}
& Z_{1}=a_{11} V_{1}, \\
& Z_{2}=a_{12} V_{1}+a_{22} V_{2}, \\
& \vdots \\
& Z_{j}=a_{1 j} V_{1}+a_{2 j} V_{2}+\cdots+a_{i j} V_{i}+\cdots+a_{j j} V_{j}, \\
& \vdots \\
& Z_{T}=a_{1 T} V_{1}+a_{2 T} V_{2}+\cdots+a_{i T} V_{i}+\cdots+a_{j T} V_{T}+\cdots+a_{T T} V_{T},
\end{aligned}
$$

where $V_{1}, \ldots, V_{T}$ are independent standard normal random variables and $a_{i j}$ is the element in the $i$ th row and $j$ th column of the matrix resulting from the Cholesky factorization in Step 3.

5. Substitute $Z_{1}, \ldots, Z_{T}$ from Step 4 into the following Taylor series-based expansion for computing the cdf, $\Phi\left(Z_{j}\right)$, of standard normal distribution (Marsaglia, 2004),

$$
\Phi\left(Z_{j}\right)=(1 / 2)+\phi\left(Z_{j}\right)\left\{Z_{j}+Z_{j}^{3} / 3+Z_{j}^{5} /(3 \cdot 5)+Z_{j}^{7} /(3 \cdot 5 \cdot 7)+\cdots\right\},
$$

where $\phi\left(Z_{j}\right)$ is the pdf of standard normal distribution, and the absolute error associated with (27) is less than $8 \times 10^{-16}$.

6. Substitute the uniform $(0,1)$ variables, $\Phi\left(Z_{j}\right)$, generated in Step 5 into the $T$ equations of the form $q_{j}\left(\Phi\left(z_{j}\right)\right)$ in (3) and (4) to generate the Burr Type III and Type XII distributions with specified values of $\xi_{3}, \xi_{4}$, and Spearman correlations.

For the purpose of demonstrating the previous steps and evaluating the proposed methodology, an example is subsequently provided to compare the MOP-based Spearman correlation procedure with the conventional MOM-based (Pearson) correlation procedure. Specifically, the distributions in Fig. 3 (dashed curves) are used as a basis of comparison using the specified correlation matrix in Table 7 where both strong and moderate correlations are considered 
in a single matrix. Let the four distributions in Fig. 3 be $Y_{1}=q_{1}\left(\Phi\left(Z_{1}\right)\right), Y_{2}=q_{2}\left(\Phi\left(Z_{2}\right)\right)$, $Y_{3}=q_{3}\left(\Phi\left(Z_{3}\right)\right)$, and $Y_{4}=q_{4}\left(\Phi\left(Z_{4}\right)\right)$, where $Y_{2}$ and $Y_{4}$ are the quantile functions from (3), and $Y_{1}$ and $Y_{3}$ are the quantile functions from (4). The specified values of MOM- and MOP-based parameters associated with these four distributions are given in Tables 3 and 4, respectively. Provided in Algorithm 2 is a source code written in Mathematica (Wolfram, 2003; Wolfram Research, 2011), which shows an example for computing intermediate correlation $\left(r_{z_{j}, z_{k}}\right)$ for the conventional product moment-based (Pearson) correlation procedure. Using Algorithm 2, all six pair-wise intermediate correlations are computed for the conventional MOM-based (Pearson) procedure, which are subsequently assembled into a $4 \times 4$ matrix. For a detailed methodology for simulating correlated Burr distributions through the method of Pearson correlation, see Headrick et al. (2010, pp. 2217-2221).

Algorithm 2. Mathematica source code for computing intermediate correlations for specified Pearson correlation procedure. The example is for Distribution $j=1$ and Distribution $k=$ $2\left(\rho_{12}\right)$. See dashed curves of Distribution 1 and Distribution 2 in Figure 3 (Panel A) and specified correlation in Table 7.

\section{(* Intermediate Correlation *)}

$r_{12}=0.868005$;

Needs[“MultivariateStatistics'”]

$f_{12}=\operatorname{PDF}\left[\right.$ MultinormalDistribution $\left.\left[\{0,0\},\left\{\left\{1, r_{12}\right\},\left\{r_{12}, 1\right\}\right\}\right],\left\{Z_{1}, Z_{2}\right\}\right]$;

$\Phi_{1}=\mathrm{CDF}\left[\right.$ NormalDistribution $\left.[0,1], Z_{1}\right]$;

$\Phi_{2}=\operatorname{CDF}\left[\right.$ NormalDistribution $\left.[0,1], Z_{2}\right]$;

(* Parameters for dashed curves of Distribution 1 and Distribution 2 in Figure 3 (Panel A) *) $c_{1}=1.097740$

$k_{1}=4.481719$;

$c_{2}=-5.542062$

$k_{2}=0.027237$

(* Quantile function from (4) and (3), respectively*)

$y_{1}=\left(\left(1-\Phi_{1}\right)^{\left(-1 / k_{1}\right)}-1\right)^{\left(1 / c_{1}\right)}$

$y_{2}=\left(\Phi_{2}^{\left(-1 / k_{2}\right)}-1\right)^{\left(1 / c_{2}\right)}$

(* Standardizing constants $\mu_{1}, \mu_{2}$ and $\sigma_{1}, \sigma_{2}$ are obtained, respectively, from equations (14) and (15) from $\left.[2]^{*}\right)$

$x_{1}=\left(y_{1}-\mu_{1}\right) / \sigma_{1}$

$x_{2}=\left(y_{2}-\mu_{2}\right) / \sigma_{2}$

(* Compute the specified conventional MOM-based (Pearson) correlation *)

$\rho_{12}=$ NIntegrate $\left[x_{1} * x_{2} * f_{12},\left\{Z_{1},-8,8\right\},\left\{Z_{2},-8,8\right\}\right.$, Method $\rightarrow$ \{“MultiDimensionalRule", "Generators" $\rightarrow$ 9\}] 
Table 8. Correlation results for the conventional MOM-based (Pearson) correlations.

\begin{tabular}{|c|c|c|c|c|}
\hline Parameter & Estimate & $95 \%$ Bootstrap Cl & Std. Error & RB\% \\
\hline & & $n=25$ & & \\
\hline$\rho_{12}=0.80$ & 0.8252 & $(0.8239,0.8266)$ & 0.00220 & 3.15 \\
\hline$\rho_{13}=0.75$ & 0.7910 & $(0.7899,0.7922)$ & 0.00155 & 5.47 \\
\hline$\rho_{14}=0.60$ & 0.6467 & $(0.6452,0.6481)$ & 0.00124 & 7.78 \\
\hline$\rho_{23}=0.70$ & 0.7125 & $(0.7110,0.7141)$ & 0.00161 & 1.79 \\
\hline$\rho_{24}=0.50$ & 0.5167 & $(0.5148,0.5185)$ & 0.00132 & 3.34 \\
\hline \multirow[t]{2}{*}{$\rho_{34}=0.40$} & 0.4117 & $(0.4094,0.4137)$ & 0.00131 & 2.93 \\
\hline & & $n=1000$ & & \\
\hline$\rho_{12}=0.80$ & 0.8026 & $(0.8025,0.8031)$ & 0.00038 & 0.33 \\
\hline$\rho_{13}=0.75$ & 0.7547 & $(0.7545,0.7551)$ & 0.00034 & 0.63 \\
\hline$\rho_{14}=0.60$ & 0.6044 & $(0.6040,0.6047)$ & 0.00026 & 0.73 \\
\hline$\rho_{23}=0.70$ & 0.7003 & $(0.7001,0.7006)$ & 0.00026 & 0.04 \\
\hline$\rho_{24}=0.50$ & 0.5007 & $(0.5004,0.5010)$ & 0.00021 & 0.14 \\
\hline$\rho_{34}=0.40$ & 0.4003 & $(0.4000,0.4006)$ & 0.00020 & - \\
\hline
\end{tabular}

Provided in Algorithm 1 is a source code written in Mathematica (Wolfram, 2003; Wolfram Research, 2011), which shows an example for computing intermediate correlation $\left(r_{z_{j}, z_{k}}\right)$ for the MOP-based Spearman correlation procedure. Using Algorithm 1, two separate matrices of corresponding intermediate correlations for the MOP-based Spearman correlation procedure are constructed for the sample sizes of $n=25$ and 1000, respectively.

The matrices of intermediate correlations can be factorized by using the Cholesky factorization. The elements of Cholesky factorization of intermediate correlation matrices are used to generate $Z_{1}, \ldots, Z_{4}$ correlated at the intermediate correlation levels by making use of formulae (26) in Step 4 with $T=4$. The values of $Z_{1}, \ldots, Z_{4}$ are then used in (27) to obtain the Taylor series-based approximations of cdfs $\Phi\left(Z_{1}\right), \Phi\left(Z_{2}\right), \Phi\left(Z_{3}\right)$, and $\Phi\left(Z_{4}\right)$, which are treated as uniform $(0,1)$ variables. These uniform variables are used in (3) and (4) to obtain quantile functions $q_{1}\left(\Phi\left(Z_{1}\right)\right), q_{2}\left(\Phi\left(Z_{2}\right)\right), q_{3}\left(\Phi\left(Z_{3}\right)\right)$, and $q_{4}\left(\Phi\left(Z_{4}\right)\right)$ to generate the four distributions in Fig. 3 that are correlated at the specified correlation level of Table 7.

For the Monte Carlo simulation, a Fortran (Microsoft Corporation, 1994.) algorithm was written for both procedures to generate 25,000 independent sample estimates for the specified parameters of (a) conventional MOM-based (Pearson) correlation $\left(\rho_{j k}\right)$, and (b) MOP-based Spearman correlation $\left(\eta_{j k}\right)$ based on samples of sizes $n=25$ and 1000 . The estimates for $\rho_{j k}$ and $\eta_{j k}$ were based on the usual formulae for the Pearson correlation and the Spearman rank correlation statistics. Bias-corrected accelerated bootstrapped Estimate, 95\% bootstrap CI, and Std. Error were obtained for the estimates associated with the parameters using 10,000 re-samples via the commercial software package Spotfire S+ (TIBCO, 2010). Further, if a parameter was outside its associated $95 \%$ bootstrap CI, then the RB\% was computed for the estimate as in (21). The results of this simulation are presented in Tables 8 and 9, and are discussed in Section 6.

\section{Discussion and conclusions}

One of the advantages of MOP-based procedure over the conventional MOM-based procedure can be expressed in the context of estimation. Inspection of Tables 5 and 6 indicates that the MOP-based estimators of left-right tail-weight ratio $\left(\xi_{3}\right)$ and tail-weight factor $\left(\xi_{4}\right)$ are 
Table 9. Correlation results for the MOP-based (Spearman) correlation procedure.

\begin{tabular}{|c|c|c|c|c|}
\hline Parameter & Estimate & $95 \%$ Bootstrap Cl & Std. Error & $\mathrm{RB} \%$ \\
\hline & & $n=25$ & & \\
\hline$\eta_{12}=0.80$ & 0.8000 & $(0.7990,0.8011)$ & 0.00053 & - \\
\hline$\eta_{13}=0.75$ & 0.7497 & $(0.7485,0.7510)$ & 0.00064 & - \\
\hline$\eta_{14}=0.60$ & 0.5980 & $(0.5961,0.5996)$ & 0.00089 & -0.33 \\
\hline$\eta_{23}=0.70$ & 0.7002 & $(0.6988,0.7017)$ & 0.00073 & - \\
\hline$\eta_{24}=0.50$ & 0.4990 & $(0.4970,0.5010)$ & 0.00101 & - \\
\hline \multirow[t]{2}{*}{$\eta_{34}=0.40$} & 0.3975 & $(0.3952,0.3996)$ & 0.00112 & -0.63 \\
\hline & & $n=1000$ & & \\
\hline$\eta_{12}=0.80$ & 0.8001 & $(0.8000,0.8003)$ & 0.00008 & - \\
\hline$\eta_{13}=0.75$ & 0.7501 & $(0.7499,0.7503)$ & 0.00010 & - \\
\hline$\eta_{14}=0.60$ & 0.6000 & $(0.5997,0.6003)$ & 0.00014 & - \\
\hline$\eta_{23}=0.70$ & 0.7001 & $(0.6999,0.7003)$ & 0.00011 & - \\
\hline$\eta_{24}=0.50$ & 0.5002 & $(0.4998,0.5004)$ & 0.00016 & - \\
\hline$\eta_{34}=0.40$ & 0.4001 & $(0.3998,0.4005)$ & 0.00017 & - \\
\hline
\end{tabular}

much less biased than the MOM-based estimators of skew $\left(\gamma_{3}\right)$ and kurtosis $\left(\gamma_{4}\right)$ when samples are drawn from the distributions with more severe departures from normality. For example, for samples of size $n=25$, the estimates of $\gamma_{3}$ and $\gamma_{4}$ for Distribution 1 (skewed and heavytailed) were, on average, $58.54 \%$ and $94.38 \%$ below their associated parameters, whereas the estimates of $\xi_{3}$ and $\xi_{4}$ were $4.98 \%$ above and $9.46 \%$ below their associated parameters. This advantage of MOP-based estimators can also be expressed by comparing their relative standard errors (RSEs), where RSE $=\{($ Std. Error/Estimate $) \times 100\}$. Comparing Tables 5 and 6 , it is evident that the estimators of $\xi_{3}$ and $\xi_{4}$ are more efficient as their RSEs are considerably smaller than the RSEs associated with the conventional MOM-based estimators of $\gamma_{3}$ and $\gamma_{4}$. For example, in terms of Distribution 1 in Fig. 3, inspection of Tables 5 and 6 (for $n=1000$ ), indicates that RSE measures of $\operatorname{RSE}\left(\hat{\xi}_{3}\right)=0.05 \%$ and $\operatorname{RSE}\left(\hat{\xi}_{4}\right)=0.03 \%$ are considerably smaller than the RSE measures of $\operatorname{RSE}\left(\hat{\gamma}_{3}\right)=0.27 \%$ and $\operatorname{RSE}\left(\hat{\gamma}_{4}\right)=0.85 \%$. This demonstrates that the estimators of $\xi_{3}$ and $\xi_{4}$ have more precision because they have less variance around their bootstrapped Estimates.

Another advantage of MOP-based procedure can be highlighted in the context of distribution fitting. In the context of fitting real-world data, the MOP-based Burr Type XII in Fig. 2 (panel B) provides a better fit to the total hospital charges data than the conventional MOMbased Burr Type XII in Fig. 2 (panel A). Comparison of the four distributions in Fig. 3 (panels A and B) clearly indicates that the MOP-based Burr Type III and Type XII distributions provide a better fit to the theoretical distributions compared with their conventional MOM-based counterparts. This advantage is most pronounced in the context of the first two distributions: Distribution 1 and Distribution 2, where the MOP-based Burr Type XII and Type III distributions (panel B) provide a better fit to the $F(3,10)$ and Chi-square $(d f=1)$ distributions than their conventional MOM-based counterparts (panel A).

Presented in Tables 8 and 9 are the simulation results of conventional MOM-based (Pearson) correlations and MOP-based Spearman correlations, respectively. Overall inspection of these tables indicates that the Spearman correlation is superior to the Pearson correlation in terms of relative bias and smaller standard errors associated with bootstrapped Estimates. For example, for $n=25$, the RB\% for the two distributions, Distribution 1 and Distribution 4, in Fig. 3 was only $-0.33 \%$ for the Spearman correlation compared with $7.78 \%$ for the Pearson correlation. It is also noted that the variability associated with the bootstrapped estimates of Spearman correlation appears to be more stable than that of the bootstrapped estimates of Pearson correlation both within and across different conditions. 
In summary, the proposed MOP-based procedure is an attractive alternative to the more traditional MOM-based procedure in the context of Burr Type III and Type XII distributions. In particular, MOP-based procedure has distinct advantages when distributions with large departures from normality are used. Finally, we note that Mathematica (Wolfram, 2003; Wolfram Research, 2011) source codes are available from the authors for implementing both conventional MOM- and MOP-based procedures.

\section{Conflict of interest}

None.

\section{References}

Abdel-Ghaly, A. A., Al-Dayian, G. R. H. Al-Kashkari, F. H. (1997). The use of Burr Type XII distributions on software reliability growth modelling. Microelectronics Reliability 37:305-313.

Burr, I. W. (1942). Cumulative frequency functions. The Annals of Mathematical Statistics 13(2):215232.

Burr, I. W. (1973). Parameters for a general system of distributions to match a grid of $\alpha_{3}$ and $\alpha_{4}$. Communications in Statistics - Theory and Methods 2(1):1-21.

Chernobai, A. S., Fabozzi, F. J., Rachev, S. T. (2007). Operational Risk: A Guide to Basel II Capital Requirements, Models, and Analysis. New York, NY: John Wiley.

Gove, J. H., Ducey, M. J., Leak, W. B., Zhang, L. (2008). Rotated sigmoid structures in managed unevenaged northern hardwood stands: A look at the Burr Type III distribution. Forestry 81:161-176.

Headrick, T. C. (2010). Statistical Simulation: Power Method Polynomials and Other Transformations. Boca Raton, FL: Chapman \& Hall/CRC.

Headrick, T. C., Pant, M. D., Sheng, Y. (2010). On simulating univariate and multivariate Burr Type III and Type XII distributions. Applied Mathematical Sciences 4:2207-2240.

Headrick, T. C. (2011). A characterization of power method transformations through $L$-moments. Journal of Probability and Statistics 2011(Article ID 497463):22 p.

Headrick, T. C., Pant, M. D. (2012). A doubling method for the generalized lambda distribution. ISRN Applied Mathematics. 2012(Article ID 725754):19 p. doi: doi:10.5402/2012/725754.

Hosking, J. R. M. (1990). L-moments: Analysis and estimation of distributions using linear combinations of order statistics. Journal of the Royal Statistical Society, Series B 52(1):105-124.

Karian, Z. A., Dudewicz, E. J. (2011). Handbook of Fitting Statistical Distributions with R. Boca Raton, FL: Chapman and Hall/CRC.

Karian, Z. A., Dudewicz, E. J. (2000). Fitting Statistical Distributions: The Generalized Lambda Distribution and Generalized Bootstrap Methods. Boca Raton, FL: Chapman and Hall/CRC.

Kendall, M., Stuart, A. (1977). The Advanced Theory of Statistics. 4th ed. New York, NY: Macmillan.

Kuo, T. C., Headrick, T. C. (2014). Simulating univariate and multivariate Tukey $g$-and- $h$ distributions based on the method of percentiles. ISRN Probability and Statistics 2014(Article ID 645823):10 p. . doi: doi:10.1155/2014/645823

Lindsay, S. R., Wood, G. R., Woollons, R. C. (1996). Modelling the diameter distribution of forest stands using the Burr distribution. Journal of Applied Statistics 23:609-619.

Marsaglia, G. (2004). Evaluating the normal distribution. Journal of Statistical Software. 11:1-10.

Mielke, P. W. (1973). Another family of distributions for describing and analyzing precipitation data. Journal of Applied Meteorology 12:275-280.

Mokhlis, N. A. (2005). Reliability of a stress-strength model with Burr Type III distributions. Communications in Statistics - Theory and Methods 34:1643-1657.

Microsoft Corporation. (1994). Microsoft FORTRAN PowerStation Version 4.0. Redmond, WA: Microsoft Corp.

Moran, P. A. P. (1948). Rank correlation and product-moment correlation. Biometrika 35:203-206.

Nadarajah, S., Kotz, S. (2006). Q exponential is a Burr distribution. Physics Letters A 359:577-579.

Nadarajah, S., Kotz, S. (2007). On the alternative to the Weibull function. Engineering Fracture Mechanics 74:451-456. 
Pant, M. D., Headrick, T. C. (2013). A method for simulating Burr Type III and Type XII distributions through $L$-moments and L-correlations. ISRN Applied Mathematics 2013(Article ID 191604):14 p. doi: doi:10.1155/2013/191604

Rodriguez, R. N. (1977). A guide to the Burr type XII distributions. Biometrika 64:129-134.

Sherrick, B. J., Garcia, P., Tirupattur, V. (1996). Recovering probabilistic information from option markets: Tests of distributional assumptions. Journal of Future Markets 16:545-560.

Tadikamalla, P. R. (1980). A look at the Burr and related distributions. International Statistical Review 48:337-344.

Tejeda, H. A., Goodwin, B. K. (2008). Modeling crop prices through a Burr distribution and analysis of correlation between crop prices and yields using a copula method. Paper presented at the Annual Meeting of the Agricultural and Applied Economics Association, Orlando, FL. . Available at: http://purl.umn.edu/6061. Accessed on Aug. 3, 2014.

TIBCO. (2010). TIBCO Spotfire S+ 8.2.0 for Microsoft Windows. Palo Alto, CA: TIBCO Software.

Wingo, D. R. (1983). Maximum likelihood methods for fitting the burr Type XII distribution to life test data. Biometrical Journal 25:77-84.

Wingo, D. R. (1993). Maximum likelihood methods for fitting the burr type XII distribution to multiply (progressively) censored life test data. Metrika 40:203-210.

Wolfram, S. (2003). The Mathematica Book. 5th ed. Champain, IL: Wolfram Media.

Wolfram Research (2011). Wolfram Mathematica 8.0.4.0 for Microsoft Windows. Champaign, IL: Wolfram Research. 